\title{
Fully Automatic Rapid inversion time (TI) Adjustment for Late Gadolinium Enhancement (LGE) Imaging Using a Pencil Beam Excitation Pulse for Single-Line T1 (SLT1) Mapping of Myocardium
}

\author{
Wolfgang G Rehwald ${ }^{1 *}$, David C Wendell ${ }^{2}$, Elizabeth Jenista ${ }^{2}$, Han W Kim², Michele Parker ${ }^{2}$, Yutaka Natsuaki ${ }^{1}$, \\ Enn-Ling Chen², Igor Klem², Raymond Kim²
}

From 19th Annual SCMR Scientific Sessions

Los Angeles, CA, USA. 27-30 January 2016

\section{Background}

LGE requires appropriate TI setting to null viable myocardium, which can be challenging and time consuming. Phase sensitive inversion recovery (PSIR) makes the TI choice less crucial for 2D imaging. However, for 3D PSIR navigator gating is required for both IR and reference data significantly increasing scan time. Additionally using a fixed single TI for a 3D scan while contrast washes out is subpotimal and worsens contrast-to-noise ratio (CNR). If repeated T1 assessment was possible during 3D LGE, scan time could be reduced and CNR optimized. Although TI scout and T1 mapping sequences could be used to determine correct TI, they cannot be executed rapidly and repeatedly during 3D LGE. We developed a rapid SLT1 technique that can be executed repeatedly during $3 \mathrm{D}$ or before 2D scans with minimal time loss. It calculates and writes the TI setting into the protocol automatically.

\section{Methods}

A pulse sequence including a single spatially non-selective IR pulse and multiple pencil beam excitation pulses $(20 \times$ $\left.20 \mathrm{~mm}, \alpha=12^{\circ}\right)$ for creating gradient echoes was implemented and tested in 17 post-contrast (POST) and 10 precontrast (PRE) free breathing patients on a 1.5T MAGNETOM Avanto (Siemens). The first echo was acquired before the IR pulse, $400 \mathrm{~ms}$ after the R-wave (Figure 1a). After the IR, data were read out every $50 \mathrm{~ms}$ for $2 \mathrm{~s}$ (POST) or 3s (PRE). The pencil beam was placed along the intersection of a mid-ventricular short-axis and the

${ }^{1}$ Siemens Healthcare, Chapel Hill, NC, USA

Full list of author information is available at the end of the article
4 chamber view, with its center in the LV cavity (Figure 1b). T1 values were calculated per pixel by a least squares trust region algorithm. Within the search region, starting at the LV center and advancing right, an edge detection algorithm found the edges between LV, septum, and RV. Myocardial T1 was calculated as average T1 in the septal compartment; blood $\mathrm{T} 1$ as average $\mathrm{T} 1$ within LV center and the septal edge. A T1 map (MAP) was acquired as gold-standard. Comparisons were made by paired t-test.

\section{Results}

Figure 2a shows a typical set of single lines acquired POST plotted versus time, showing septum and blood pools. Their different $\mathrm{T} 1$ recovery and septal motion can be seen. By SLT1 and MAP respectively, POST myocardial $\mathrm{T} 1$ (mean $\pm \mathrm{SD}$, ms) was $415 \pm 127$ and $409 \pm 125$ and POST blood T1 was $176 \pm 100$ and $296 \pm 117$. T1 was identical $(\mathrm{p}>0.05)$ in both myocardium and blood. PRE myocardial T1 was identical by SLT1 and MAP respectively $(1031 \pm 68$ and $1039 \pm 29$, $\mathrm{p}>0.05)$, whereas blood T1 was not $(1488 \pm 172$ and $1641 \pm 107, \mathrm{p}<0.05)$. Figure $2 \mathrm{~b}$ shows POST T1 values of myocardium (top) and blood (bottom) for all patients. The matching value pairs (left graphs) illustrate the similarity of SLT1 and MAP values. The graphs on the right show the close correlation of SLT1 and MAP data.

\section{Conclusions}

This simple, rapid, and fully automated adjustment technique can be used to calculate myocardial T1 and set the optimal myocardial TI for LGE scans. It allows 

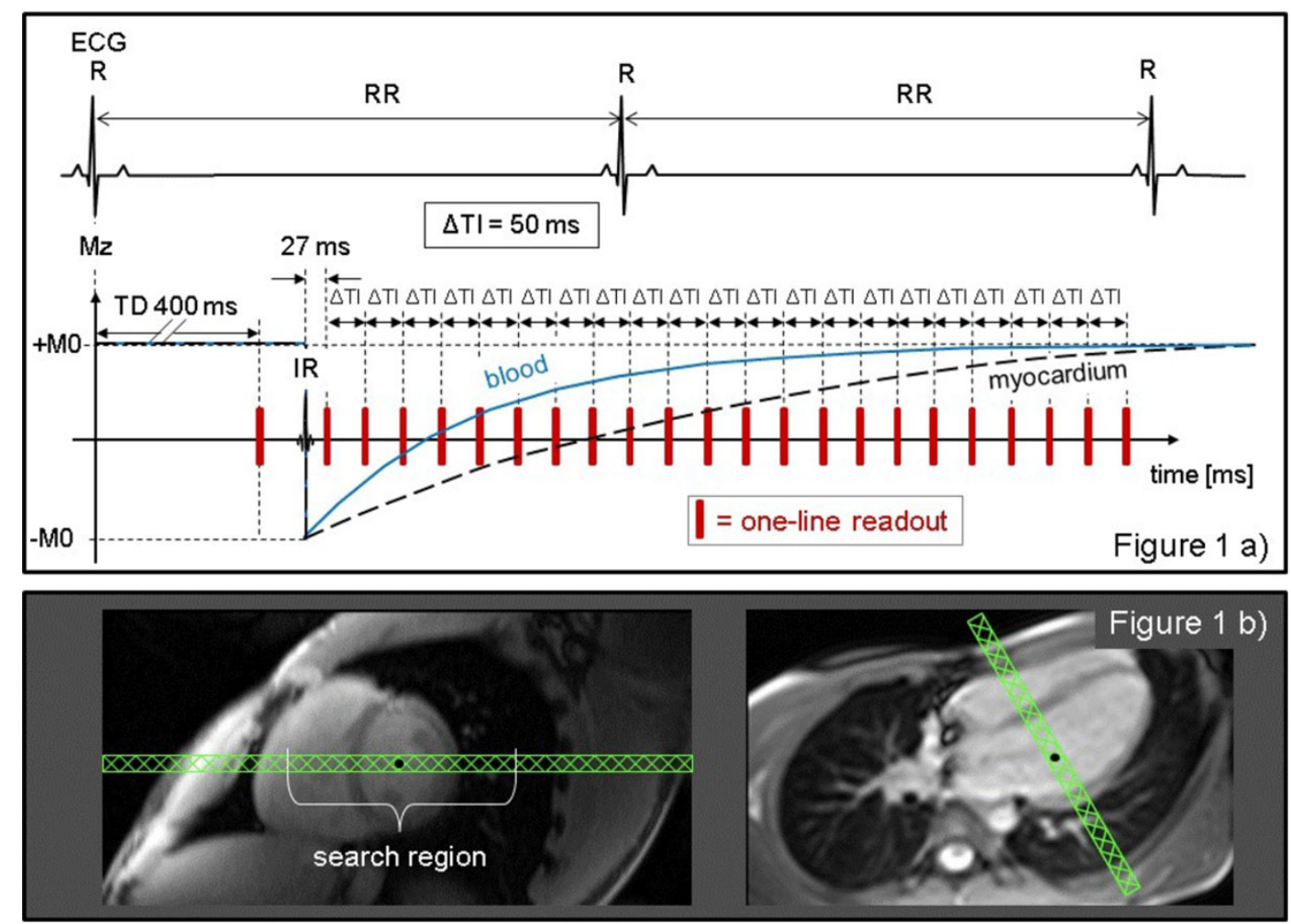

Figure 1
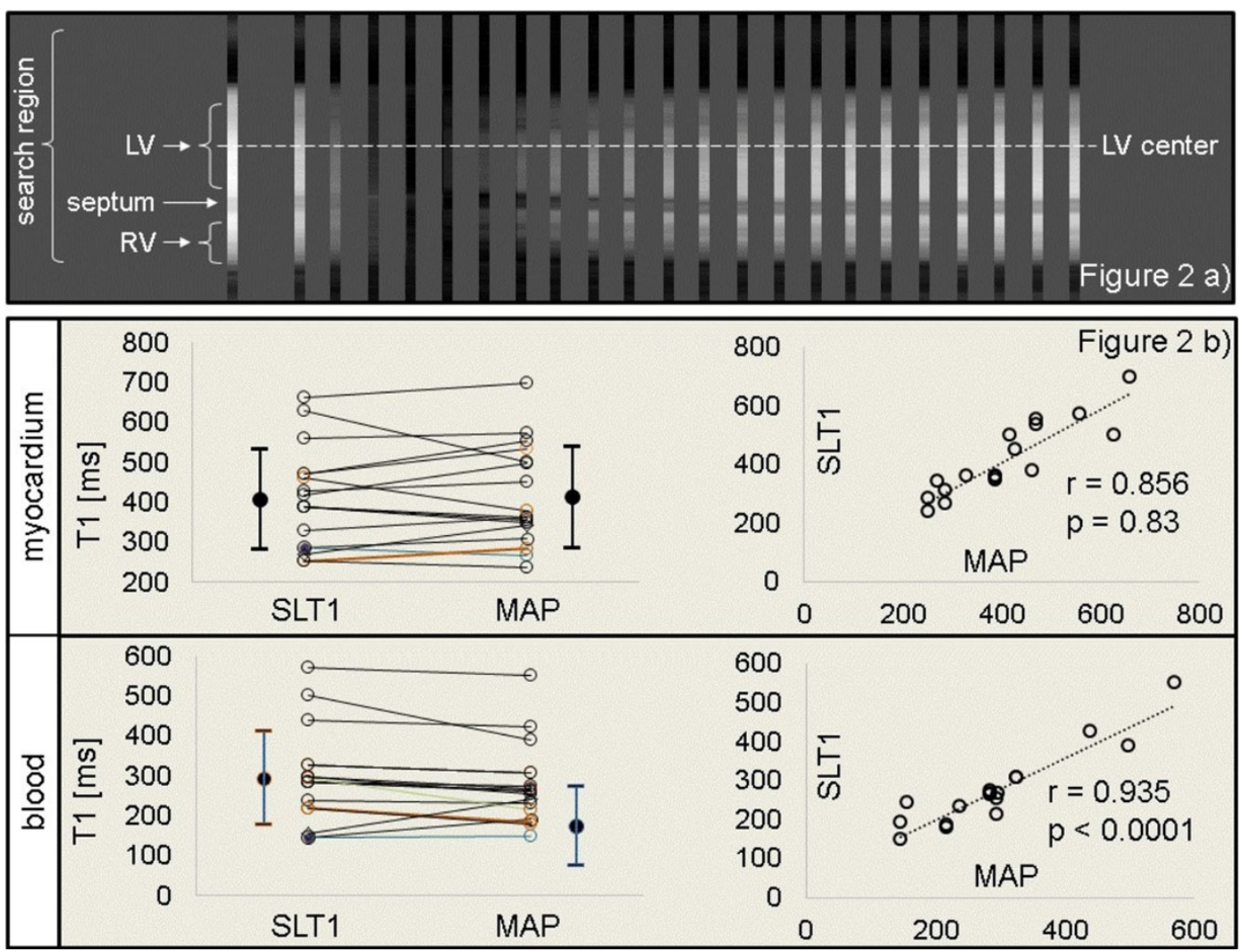

Figure 2 
quick TI adjustment for 2D and readjustment during 3D scans. The technique is repeatable and the beam can be placed automatically without operator intervention.

\section{Authors' details}

${ }^{1}$ Siemens Healthcare, Chapel Hill, NC, USA. ²Duke Cardiovascular MR Center,

Duke University Medical Center, Durham, NC, USA.

Published: 27 January 2016

doi:10.1186/1532-429X-18-S1-P322

Cite this article as: Rehwald et al:: Fully Automatic Rapid inversion time

(TI) Adjustment for Late Gadolinium Enhancement (LGE) Imaging Using a

Pencil Beam Excitation Pulse for Single-Line T1 (SLT1) Mapping of

Myocardium. Journal of Cardiovascular Magnetic Resonance 2016 18(Suppl 1):

P322.

Submit your next manuscript to BioMed Central and take full advantage of:

- Convenient online submission

- Thorough peer review

- No space constraints or color figure charges

- Immediate publication on acceptance

- Inclusion in PubMed, CAS, Scopus and Google Scholar

- Research which is freely available for redistribution 